\title{
BMJ Open Venous thromboembolism incidence in the Ireland east hospital group: a retrospective 22-month observational study
}

\author{
Barry Kevane, ${ }^{1,2}$ Mary Day, ${ }^{2}$ Noirin Bannon, ${ }^{2}$ Leo Lawler, ${ }^{2,3}$ Tomas Breslin, ${ }^{2,4}$ \\ Claire Andrews, ${ }^{1,2}$ Howard Johnson, ${ }^{2,5}$ Michael Fitzpatrick, ${ }^{2}$ Karen Murphy, ${ }^{2,6}$ \\ Olivia Mason, ${ }^{7}$ Annemarie O'Neill, ${ }^{2,8}$ Fionnuala Donohue,${ }^{5}$ Fionnuala Ní Áinle ${ }^{1,2,9}$
}

To cite: Kevane B, Day M, Bannon N, et al. Venous thromboembolism incidence in the Ireland east hospital group: a retrospective 22-month observational study. BMJ Open 2019;9:e030059. doi:10.1136/ bmjopen-2019-030059

- Prepublication history and additional material for this paper are available online. To view please visit the journal (http:// dx.doi.org/10.1136/bmjopen2019-030059).

Received 25 February 2019

Revised 2 May 2019

Accepted 12 June 2019
Check for updates

(C) Author(s) (or their employer(s)) 2019. Re-use permitted under CC BY-NC. No commercial re-use. See rights and permissions. Published by BMJ.

For numbered affiliations see end of article.

Correspondence to Professor Fionnuala Ní Áinle; fniainle@mater.ie

\section{ABSTRACT}

Objectives To determine the incidence of venous thromboembolism (VTE) and the incidence of hospitalacquired VTE (HA-VTE) arising within the population served by the Ireland East Hospital Group (IEHG).

Design A retrospective observational study was conducted using hospital discharge data obtained from the hospital inpatient enquiry data reporting system. In this system, VTE events recorded as 'primary diagnosis' represented the reason for initial hospital admission, whereas VTE recorded as a 'secondary diagnosis' occurred following admission and were therefore used as an approximation of HA-VTE. These data were used to estimate the overall incidence of VTE and the proportion of these events which were hospital-acquired.

Setting The IEHG is the largest hospital group in the Irish healthcare system and serves a population of over 1 million individuals.

Participants Data were generated from records pertaining to the 2727 patient admission episodes where a diagnosis of VTE was made during the 22-month study period.

Results During the study period, 2727 VTE events were recorded within the IEHG (which serves a population of 1036279 ) corresponding to an incidence of 1.44 (95\% Cl 1.36 to 1.51) per 1000 per annum. 1273 (47\%) of VTE events were recorded as secondary VTE. The incidence of VTE was highest among individuals over 85 years of age (16.03 per $1000 ; 95 \% \mathrm{Cl} 12.81$ to 19.26 ) and was more common following emergency hospital admission. Conclusion These data suggest that HA-VTE accounts for at least $47 \%$ of all VTE events arising within a hospital group serving a population of over 1 million individuals within the Ireland. Given that HA-VTE is a well-recognised source of (potentially preventable) hospital deaths, these findings provide a compelling argument for prioritising strategies directed at reducing the risk of VTE among hospital patients served by the IEHG and within the Ireland as a whole.

\section{INTRODUCTION}

Venous thromboembolism (VTE) comprises deep vein thrombosis (DVT) and pulmonary
Strengths and limitations of this study

- This study represents the first instance in which data pertaining to venous thromboembolism (VTE) incidence within the Ireland have been reported from data systematically collected from hospital discharge records within the largest hospital group in the Ireland.

- Data were collected from large academic centres as well as smaller community hospitals and the National Maternity Hospital.

- The data collection methods utilised were not able to accurately identify all hospital-acquired VTE (HAVTE) cases occurring in the community (but within 90 days of a hospital admission), highlighting the urgent need to develop systems to accurately measure HA-VTE in Irish Healthcare.

embolism (PE) and is a major contributor to global disease burden, affecting millions of individuals worldwide every year. ${ }^{1}$ VTE is the third the most common cause of death from cardiovascular disease after heart

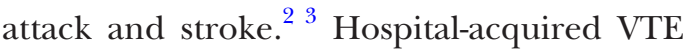
(HA-VTE, defined as a VTE event occurring either during or in the 90 days following hospitalisation) is an important source of death and disability with up to $50 \%-60 \%$ of all VTE cases occurring during or after hospitalisation, making it a leading preventable cause of hospital death. ${ }^{14-7}$ In the UK, upwards of 25000 cases of HA-VTE occurred every year prior to the implementation of a national programme aimed at reducing HA-VTE. ${ }^{8}$ These and other data demonstrate that HA-VTE is potentially preventable with risk assessment and implementation of appropriate thromboprophylaxis. ${ }^{4-6}$

VTE diagnosis, treatment and follow-up require well-structured multidisciplinary pathways with clear associated guidelines. 


\begin{tabular}{lcccccccc} 
Table 1 & \multicolumn{2}{l}{ Distribution and characteristics of VTE events by hospital } \\
Hospital & Total VTE & Primary (\%) & Secondary (\%) & Emergency (\%) & Elective (\%) & Maternity (\%) & Male (\%) & Female (\%) \\
\hline MMUH & 674 & $245(36.4)$ & $429(63.6)$ & $589(87.4)$ & $84(12.5)$ & $1(0.1)$ & $343(50.9)$ & $331(49.1)$ \\
SVUH & 946 & $502(53)$ & $444(47)$ & $818(86.5)$ & $107(11.3)$ & $21(2.2)$ & $450(47.6)$ & $496(52.4)$ \\
SLGH & 204 & $142(69.6)$ & $62(30.4)$ & $185(90.7)$ & $10(4.9)$ & $9(4.4)$ & $89(43.6)$ & $115(56.4)$ \\
WGH & 220 & $151(68.6)$ & $69(31.4)$ & $200(90.9)$ & $7(3.2)$ & $13(5.9)$ & $93(42.3)$ & $127(57.7)$ \\
MRH & 200 & $117(58.5)$ & $83(41.5)$ & $165(82.5)$ & $7(3.5)$ & $28(14)$ & $83(41.5)$ & $117(58.5)$ \\
OLH & 156 & $76(48.7)$ & $80(51.2)$ & $131(84)$ & $25(16)$ & $0(0)$ & $80(51.3)$ & $76(48.7)$ \\
SMH & 29 & $23(79.3)$ & $6(20.7)$ & $28(96.6)$ & $1(3.4)$ & $0(0)$ & $14(48.3)$ & $15(51.7)$ \\
SCH & 209 & $189(90.4)$ & $20(9.6)$ & $201(96.2)$ & $8(3.8)$ & $0(0)$ & $72(34.4)$ & $137(65.6)$ \\
CNOH & 28 & $1(3.6)$ & $27(96.4)$ & $1(3.6)$ & $27(96.4)$ & $0(0)$ & $5(17.9)$ & $23(82.1)$ \\
RVEEH & 3 & $1(33.3)$ & $2(66.6)$ & $0(0)$ & $3(100)$ & $0(0)$ & $1(33.3)$ & $2(66.6)$ \\
NMH & 58 & $7(12.1)$ & $51(87.9)$ & $1(1.7)$ & $1(1.7)$ & $56(96.6)$ & $1(1.7)$ & $57(98.3)$ \\
\hline
\end{tabular}

$\mathrm{CNOH}$, Cappagh National Orthopaedic Hospital; MMUH, Mater Misericordiae University hospital; MRH, Midlands Regional Hospital; $\mathrm{NMH}$, National Maternity Hospital; OLH, Our Female's Hospital Navan; RVEEH, Royal Victoria Eye and Ear Hospital; SCH, St Columcille's Hospital; SLGH, St Luke's General Hospital; SMH, St Michael's Hospital; SVUH, St Vincent's University Hospital; VTE, venous thromboembolism; WGH, Wexford General Hospital.

Consequently, detailed knowledge of VTE incidence and mortality in a healthcare system is of critical importance when planning and resourcing VTE care. Despite the well-characterised contribution of VTE to disease burden internationally, Irish data are lacking and VTE awareness is poor. This has the potential to translate into clinical risk, due to poor resourcing and planning of VTE prevention, diagnosis and treatment. Overall, a VTE incidence of $\sim 100$ per 100000 person-years is reported in developed countries. ${ }^{9}{ }^{10}$ However, accurate characterisation of the HA-VTE burden in Ireland is not currently possible as these events are not specifically captured by current hospital coding mechanisms.

Irish Health Care is organised into seven Hospital Groups. The Ireland East Hospital Group (IEHG) encompasses 11 hospitals (table 1) and serves a population of 1036 279. A group-wide oversight on VTE across the IEHG has recently deemed a priority and an IEHG VTE committee was established in May 2017 to address prevention, diagnosis and treatment of this important condition. Given the lack of data on VTE incidence within the group and the consequent challenges in planning care as outlined above, an early identified action was to assess the number of VTE events in IEHG and to estimate the contribution of HA-VTE to overall VTE burden. The aim of this study was to retrospectively assess and characterise VTE events in IEHG during a 22-month period.

\section{Patient and public involvement}

Thrombosis Ireland (the only patient advocacy group in the Ireland dedicated to promoting awareness of VTE risk) is represented on the IEHG VTE working group. This organisation has strongly advocated for the improved collection and dissemination of data relating to VTE risk in Ireland in order to enhance public awareness of this risk and contributed directly to the concept and conduct of this study.

\section{METHODS}

This was a retrospective observational study during a 22-month prespecified period (January 2016-October 2017) during which time there were 600442 inpatient and day case discharges from the IEHG hospitals.

\section{The study population}

The study population at outset comprised the population served by the 11 hospitals of the IEHG. The estimated population of the IEHG indicative catchment area (based on census data collected during the prespecified study period) is 1036279 (source: HealthAtlas Ireland, Health Service Executive (HSE) of Ireland).

\section{Data source}

The data source for this study comprised the consolidated hospital inpatient enquiry records (HIPE; a commonly used data source in Irish hospitals) of the 11 IEHG hospitals.

HIPE data are entered and validated by trained HIPE coders at hospital level at the time of hospital discharge, with the data extracted from standardised discharge forms completed by the responsible discharging clinician. After entry of data by HIPE coders at the hospital level, it is further validated by the centralised HSE HIPE Healthcare Pricing Office when uploaded there from each hospital. For the purposes of this study, datasets generated through the consolidation of HIPE data by National Quality Assurance Intelligence System (NQAIS) Clinical were analysed. NQAIS Clinical is a reporting tool which was developed by the Health Intelligence Unit of the HSE and analyses HIPE data for patient discharges following their episode of care. This system was designed collaboratively by the HSE and nominated hospital group representatives and developed by OpenApp (Dublin, Ireland) and is deployed under the governance framework of the HSE. HealthAtlas Ireland (a software system developed in 
collaboration with the HSE) organised the extract of VTE datasets from NQAIS.

\section{VTE case identification}

We identified VTE cases on the basis of International Classification of Disease, Tenth Revision (ICD-10) discharge diagnostic codes for VTE (online supplementary table). Diagnosis of VTE within the IEHG is made in accordance with the current UK National Institute for Health and Care Excellence Guidelines. For the purposes of this study, the committee deliberated but ultimately chose to include codes pertaining to superficial vein thrombophlebitis and obstetric embolism, to address the possibility that VTE events may have been coded under these ICD codes. However, these diagnoses accounted for very few reported VTE events overall (63 cases in total) and their exclusion from the final analysis would not have impacted significantly on the overall VTE incidence. In NQAIS, the term 'Primary Diagnosis' refers to the primary assigned diagnosis on presentation to a hospital, that is the reason the patient was admitted to hospital. The term 'Subsequent (secondary) Diagnoses' refer to diagnoses arising during hospitalisation (ie, not the reason for initial hospital admission). Consequently, 'Subsequent (secondary) Diagnoses' data can be considered to represent a surrogate marker for HA-VTE. VTE events managed via an ambulatory care pathway in the emergency department (and therefore not admitted) were not captured.

\section{Data analysis}

IEHG data analysts analysed datasets generated by NQAIS Clinical in spreadsheet form, filtering data by VTE ICD code and by the primary or secondary diagnosis as well as other parameters such as age, gender and so on. Descriptive statistics describing VTE incidence patterns were generated and the relationship between variables was summarised by correlation and crosstabulation analysis after importing data to SPSS V.24.

\section{Ethical approval}

As our results were generated from existing datasets populated with anonymised data and maintained by the HSE (where individual patients were not identifiable to us), ethical approval was not required to proceed with this study.

\section{RESULTS}

During this prespecified 22-month time period (January 2016-October 2017), 2727 VTE events were recorded (according to the identified ICD-10 codes and excluding elective day cases). The majority (59.4\%) of these cases were diagnosed in the two large Dublin teaching hospitals (MMUH and SVUH) with smaller numbers identified in the remaining general hospitals that serve areas outside the catchment areas of these two large academic centres (table 1). Based on census data which describe the population size within the individual hospital and hospital group catchment areas (total IEHG catchment area population: 1036 279), these data suggest a VTE incidence of 1.44 (95\% CI 1.36 to 1.51 ) per 1000 per annum. In order to characterise the spread of VTE events by patient age, an analysis was performed in which VTE events in the age categories $<18,18-25,26-45,46-65,66-85$ and $>85$ years were determined (table 2 ). The majority (37.6\%) of VTE events recorded during the 22-month study period were observed in the 66-85year age category. Using population data based on the 2016 census report, the age-specific incidence of VTE was also determined, with the highest incidence rate observed, predictably, within the $>85$ years age category (16.03 per 1000 ; $95 \%$ CI 12.8 to 19.26 ) and with increasing median age correlating directly with increasing VTE incidence within these age groups $(\mathrm{r}=1.0$, $\mathrm{p}=0.002$; Spearman $r$ correlation coefficient).

A total of $1273(47 \%)$ VTE cases were 'secondary' diagnoses and 1454 (53\%) were 'primary' diagnoses (figure 1A). As discussed in the 'Methods' section, the term 'secondary' VTE diagnosis is an approximation (but a likely significant underestimation) of HA-VTE. The proportion of VTE cases representing a 'secondary' diagnosis varied considerably across IEHG hospitals (table 1) from $47 \%$ and $63.6 \%$ in SVUH and MMUH respectively to $9.6 \%$ in SCH. The majority $(89.2 \%)$ of all VTE events (primary and secondary combined and excluding maternity admissions) occurred in the context of emergency admission to hospital (figure 1B). Predictably, the number

Table 2 Age-specific incidence of VTE within the IEHG

\begin{tabular}{lcccccc}
\hline & & & & & Annual \\
Age (years) & Total (\%) & Male (\%) & Female (\%) & 95\% Cl & \\
\cline { 6 - 7 } & $4(0.15)$ & $1(0.08)$ & $3(0.2)$ & 0.00 & 0.00 & 0.01 \\
\hline 18 & $76(2.8)$ & $24(1.9)$ & $52(3.5)$ & 0.74 & 0.51 & 0.96 \\
\hline $26-45$ & $599(21.9)$ & $258(20.9)$ & $341(22.8)$ & 1.87 & 1.67 & 2.08 \\
$46-65$ & $853(31.3)$ & $443(35.9)$ & $410(27.4)$ & 4.21 & 3.83 & 4.59 \\
$66-85$ & $1026(37.6)$ & $449(36.4)$ & $577(38.5)$ & 10.50 & 9.64 & 11.37 \\
$>85$ & $171(6.3)$ & $57(4.62)$ & $114(7.6)$ & 16.03 & 12.81 & 19.26 \\
\hline
\end{tabular}

IEHG, Ireland East Hospital Group; VTE, venous thromboembolism. 
A

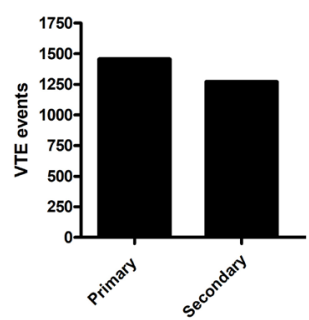

Figure 1 The majority of VTE events reported were primary events and occurred most frequently in the setting of an emergency hospital admission. The majority of reported VTE events were primary with secondary VTE accounting for $47 \%$ of total reported events (A). When VTE events were assessed by admission-type, the majority were found to have occurred in the context of an emergency admission (B). VTE, venous thromboembolism.

of VTE cases reported in hospitals providing specialist surgical or obstetric services as opposed to general acute medical care (such as the Cappagh National Orthopaedic Hospital, the National Maternity Hospital and the Royal Victoria Eye and Ear Hospital) was lower than that seen in the other centres and primarily comprised HA-VTE events (table 1 ).

The two large urban hospitals, which recorded the majority of VTE events overall, serve two distinct populations with contrasting socioeconomic profiles as evidenced by the greater degree of social deprivation affecting the catchment area of the MMUH relative to the more affluent population served by the SVUH (Census 2016, Central Statistics Office, Ireland). A crosstabulation of VTE incidence by age and gender in these two populations did not demonstrate any significant difference in VTE incidence ( $\mathrm{p}=0.2$; Fisher exact test). Moreover, no differences in VTE incidence by age or gender in these contrasting populations was seen when VTE events were analysed in the categories of 'primary' or 'secondary' VTE diagnosis specifically (primary VTE, $\mathrm{p}=0.3$; secondary VTE, $p=0.6$; Fisher exact test).

Within the subcategory of secondary VTE events specifically, $77.2 \%$ occurred in the context of an emergency admission with the remainder occurring following an elective hospital admission. Among the individuals who developed a VTE as a secondary diagnosis, the most frequently recorded primary diagnoses (leading to the initial admission to hospital) prior to VTE diagnosis were cancer $(16.4 \%)$, respiratory disease $(14.6 \%)$ and cardiovascular disease (13.5\%) (figure 2 ).

\section{DISCUSSION}

In this study, we report and characterise VTE events during a 22-month period in an entire Irish Hospital Group. This report represents the first time that comprehensive VTE data have been available pertaining to an Irish hospital group and represents the first step towards improved capture and auditing of VTE data at a national

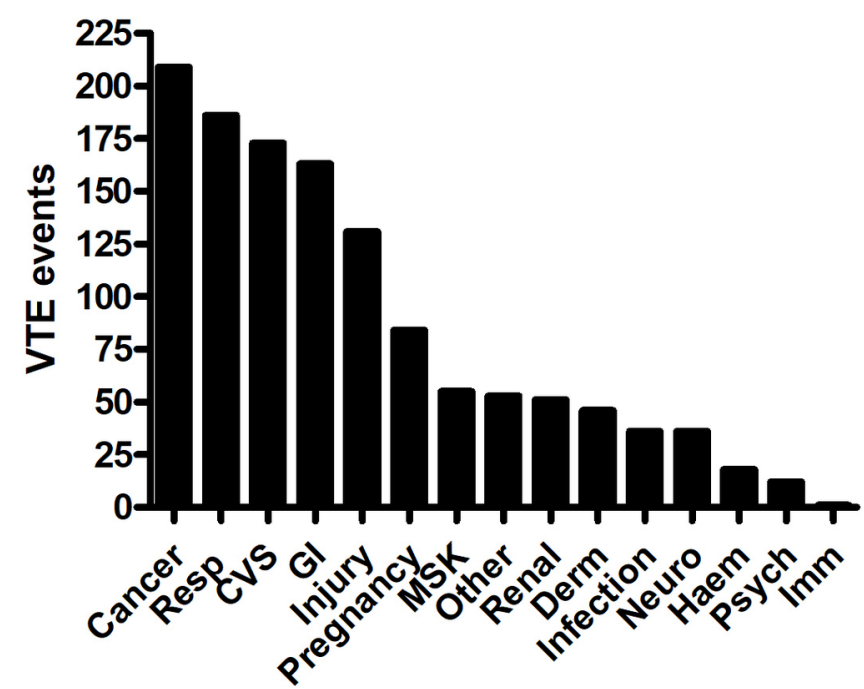

Figure 2 Distribution of secondary VTE events according to initial admission diagnosis Among the cases of VTE which were reported as occurring during hospital admission, the most frequently recorded primary causes of admission were cancer followed by resp, CVS and GI. CVS, cardiovascular disease; derm, dermatological; Gl, gastrointestinal disease; haem, haematological; imm, immunological; MSK, musculoskeletal; neuro, neurological; psych, psychiatric; resp, respiratory disease; VTE, venous thromboembolism.

level. During this prespecified 22-month time period (January 2016-October 2017), 2727 VTE events were recorded, and critically, almost half were 'secondary' VTE diagnoses.

The coded diagnosis of 'secondary' VTE was used as an approximation of HA-VTE for the purposes of this study, reflecting the methodology of current hospital data collection systems in Ireland. This most likely led to an underestimation of the total burden of HA-VTE in our study population given the recent findings that a significant majority of HA-VTE events occur after hospital discharge (and as our data collection systems would likely code these particular events as 'primary'). ${ }^{9}$

On a global scale, VTE is a serious and common healthcare problem. A total of 544000 VTE-related deaths occur annually in Europe. In other words, VTE is responsible for more deaths than AIDS, breast cancer, prostate cancer and motor vehicle accidents combined..$^{10}$ Moreover, VTE causes major morbidity in survivors due to lifelong debilitating chronic conditions. Approximately 400000 individuals in Europe experience chronic thromboembolic pulmonary hypertension and post-thrombotic syndrome (characterised by lower limb swelling, pain, varicose veins and ulceration) every year. ${ }^{10}$ VTE associated with hospitalisation is the leading contributor to disability-adjusted life years in low-income and middle-income countries and is the second leading contributor in the developed world. ${ }^{1}$ The economic impact of VTE is enormous: it is estimated that VTE is associated with annual costs of $€ 640$ million per year in the UK National Health Service. ${ }^{8}$ Irish data are currently not available. 
Given that a significant proportion of VTE events are fatal, it is likely that HA-VTE is, therefore, an important cause of potentially preventable hospital death. ${ }^{4}$ In the UK, the House of Commons Health Committee reported in 2005 that an estimated 25000 people die from preventable HA-VTE every year. ${ }^{8}$ The relative risk of VTE has been reported to increase $>100$-fold during and after hospitalisation compared with community rates. ${ }^{911}$ Randomised clinical trials have shown that pharmacological thromboprophylaxis can reduce the incidence of PE and DVT in medical and surgical patients. ${ }^{12-14}$ However, universal thromboprophylaxis cannot be recommended due to competing bleeding risks. ${ }^{14}{ }^{15}$ Therefore, risk assessment of hospitalised patients to identify those with an elevated VTE risk and a low bleeding risk is essential, followed by targeted appropriate thromboprophylaxis. ${ }^{4} 1214$ A UK national quality initiative to increase the number of hospitalised patients assessed for risk of VTE, based on UK National Institute for Health and Care Excellence recommendations ${ }^{4}$ has resulted in a significant reduction in VTE mortality. ${ }^{5}$ Anticoagulant thromboprophylaxis has also been reported to significantly reduce fatal PE in medical patients with significant VTE risk factors in other developed countries. ${ }^{14} 16-18$ However, recently published data suggest that limiting the duration of thromboprophylaxis to the short period of hospitalisation may not be sufficient for at-risk medical patients ${ }^{9}$ and extended prophylaxis requires further investigation in this cohort. ${ }^{19}$

Consequently, the findings of the current study have enormous potential implications for Irish healthcare. Unlike in the UK, VTE risk assessment is not mandatory for hospitalised patients in Ireland. Some individual hospitals have reported excellent compliance with guidelines mandating VTE risk assessment and implementation of appropriate thromboprophylaxis ${ }^{20}$ however, other published data suggest poor compliance. ${ }^{21}$

Although this study represents the first instance in which the burden of VTE, and in particular HA-VTE, in the Ireland has been estimated, the strength of our findings is somewhat weakened by the limitations which currently exist in Irish data collection systems with respect to the detailed characterisation of VTE events as outlined above. In particular, it is not currently possible to definitively identify every individual case of HA-VTE using HIPE data given the inability of current measures to capture HA-VTE occurring postdischarge. Similarly, it is not possible to account for patients with VTE from within the catchment of the IEHG who may have presented to other hospitals (and vice versa) and it is possible that there may be some duplication in recorded events as individual patient identifiers were not collected in these datasets (all data were anonymised and individual patient episodes were analysed as opposed to individual patients). In addition, our datasets were not populated with additional potentially significant information, such as whether patients were admitted to surgical or medical wards and so on and patients managed on ambulatory pathways without hospital admission would not have been reported to HIPE.

Robust and reliable data collection processes are clearly crucial to quality improvement initiatives and particularly for promoting increased understanding of potential health risks (such as those posed by insufficient awareness of the burden of HA-VTE). Therefore, the weaknesses in our methodology clearly highlight the urgent need to develop data collecting tools which will accurately characterise HA-VTE events in a manner that will inform future policy with respect to the introduction and implementation of mandatory VTE risk reduction protocols, targeting of particularly high-risk patient sub-groups and so on. It is likely that in the future, the more widespread use of electronic health records and the unique patient identifier will improve the accuracy of data collection, although currently, electronic health records are not in widespread use across hospitals in this country.

In conclusion, notwithstanding the potential limitations, as outlined above, the results of the present study (suggesting that at least $47 \%$ of VTE diagnosed within the IEHG are hospital-acquired events) provides a compelling argument for the urgent implementation of strategies targeting this important source of preventable morbidity and mortality in our hospitals.

\section{Author affiliations}

${ }^{1}$ Department of Haematology, Mater Misericordiae University Hospital, Dublin, Ireland

${ }^{2}$ Ireland East Hospital Group, Dublin, Ireland

${ }^{3}$ Department of Radiology, Mater Misericordiae University Hospital, Dublin, Ireland

${ }^{4}$ Department of Emergency Medicine, Mater Misericordiae University Hospital, Dublin, Ireland

${ }^{5}$ Health Intelligence Unit, R\&D, Health Service Executive, Dublin, Ireland ${ }^{6}$ Department of Haematology, St Vincent's University Hospital, Dublin, Ireland ${ }^{7}$ Centre for Support and Training in Analysis and Research (CSTAR), University College Dublin, Dublin, Ireland

${ }^{8}$ Thrombosis Ireland, Dublin, Ireland

${ }^{9}$ Irish Network for VTE Research (INViTE)

Acknowledgements The authors are honoured that the founder of the patient organization Thrombosis Ireland (and co-author, AON) sits on IEHG VTE committee.

Contributors BK: performed data and statistical analyses and wrote the manuscript. MD, KM and AON: development of the concept of the study and drafting of the final manuscript. NB, LL and TB: reviewed and edited the final manuscript. CA: data analysis and preparation of the manuscript. HJ and MF: concept of the study, data and statistical analyses, and reviewed and edited the final manuscript. OM: statistical analysis. FD: data collection and analysis. FNA: developed the concept of the study, contributed to writing the manuscript, data analysis and edited the final manuscript.

Funding The authors have not declared a specific grant for this research from any funding agency in the public, commercial or not-for-profit sectors.

Competing interests FNA has received research funding from Leo Pharma and Bayer however these awards are not relevant to the current study.

Patient consent for publication Not required.

Provenance and peer review Not commissioned; externally peer reviewed.

Data sharing statement All data relevant to the study are included in the article or uploaded as supplementary information.

Open access This is an open access article distributed in accordance with the Creative Commons Attribution Non Commercial (CC BY-NC 4.0) license, which permits others to distribute, remix, adapt, build upon this work non-commercially, and license their derivative works on different terms, provided the original work is 
properly cited, appropriate credit is given, any changes made indicated, and the use is non-commercial. See: http://creativecommons.org/licenses/by-nc/4.0/.

\section{REFERENCES}

1. ISTH Steering Committee for World Thrombosis Day. Thrombosis: a major contributor to the global disease burden. $J$ Thromb Haemost 2014;12:1580-90.

2. Anderson FA, Wheeler HB, Goldberg RJ, et al. A population-based perspective of the hospital incidence and case-fatality rates of deep vein thrombosis and pulmonary embolism. The Worcester DVT Study. Arch Intern Med 1991;151:933-8.

3. Naess IA, Christiansen SC, Romundstad P, et al. Incidence and mortality of venous thrombosis: a population-based study. $J$ Thromb Haemost 2007;5:692-9.

4. National Institute for Health and Care Excellence (NICE) clinical guideline CG92. Venous thromboembolism: reducing the risk for patients in hospital. https://www.nice.org.uk/guidance/cg92.

5. Catterick D, Hunt BJ. Impact of the national venous thromboembolism risk assessment tool in secondary care in England: retrospective population-based database study. Blood Coagul Fibrinolysis 2014;25:571-6.

6. Lester W, Freemantle N, Begaj I, et al. Fatal venous thromboembolism associated with hospital admission: a cohort study to assess the impact of a national risk assessment target. Heart 2013;99:1734-9.

7. Jha AK, Larizgoitia I, Audera-Lopez C, et al. The global burden of unsafe medical care: analytic modelling of observational studies. BMJ Qual Saf 2013;22:809-15.

8. Parliament.UK. House of Commons Health Committee Report on the Prevention of Venous Thromboembolism in Hospitalised Patients. www.publications.parliament.uk/pa/cm200405/cmselect/cmhealth/ 99/9902.html.

9. Heit JA, Crusan DJ, Ashrani AA, et al. Effect of a near-universal hospitalization-based prophylaxis regimen on annual number of venous thromboembolism events in the US. Blood 2017;130:109-14.

10. Cohen AT, Agnelli G, Anderson FA, et al. Venous thromboembolism (VTE) in Europe. The number of VTE events and associated morbidity and mortality. Thromb Haemost 2007;98:756-64.

11. Sweetland S, Green J, Liu B, et al. Duration and magnitude of the postoperative risk of venous thromboembolism in middle aged women: prospective cohort study. BMJ 2009;339:b4583.
12. Gould MK, Garcia DA, Wren SM, et al. Prevention of VTE in nonorthopedic surgical patients: Antithrombotic Therapy and Prevention of Thrombosis, 9th ed: American College of Chest Physicians Evidence-Based Clinical Practice Guidelines. Chest 2012;141(2 Suppl):e227S.

13. Falck-Ytter Y, Francis CW, Johanson NA, et al. Prevention of VTE in orthopedic surgery patients: Antithrombotic Therapy and Prevention of Thrombosis, 9th ed: American College of Chest Physicians Evidence-Based Clinical Practice Guidelines. Chest 2012;141(2 Suppl):e278S-325

14. Kahn SR, Lim W, Dunn AS, et al. Prevention of VTE in nonsurgica patients: Antithrombotic Therapy and Prevention of Thrombosis, 9th ed: American College of Chest Physicians Evidence-Based Clinical Practice Guidelines. Chest 2012;141(2 Suppl):e195S-226.

15. Le P, Martinez KA, Pappas MA, et al. A decision model to estimate a risk threshold for venous thromboembolism prophylaxis in hospitalized medical patients. J Thromb Haemost 2017;15:1132-41.

16. Alikhan R, Cohen AT. Heparin for the prevention of venous thromboembolism in general medical patients (excluding stroke and myocardial infarction). Cochrane Database Syst Rev 2009;3:CD003747.

17. Dentali F, Douketis JD, Gianni M, et al. Meta-analysis: anticoagulant prophylaxis to prevent symptomatic venous thromboembolism in hospitalized medical patients. Ann Intern Med 2007;146:278-88.

18. Lloyd NS, Douketis JD, Moinuddin I, et al. Anticoagulant prophylaxis to prevent asymptomatic deep vein thrombosis in hospitalized medical patients: a systematic review and meta-analysis. J Thromb Haemost 2008;6:405-14.

19. Khalafallah AA, Kirkby BE, Wong S, et al. Venous thromboembolism in medical patients during hospitalisation and 3 months after hospitalisation: a prospective observational study. BMJ Open 2016;6:e012346.

20. O'Shaughnessy F, Donnelly JC, Cooley SM, et al. Thrombocalc: implementation and uptake of personalized postpartum venous thromboembolism risk assessment in a high-throughput obstetric environment. Acta Obstet Gynecol Scand 2017;96:1382-90.

21. Adamali $\mathrm{H}$, Suliman $\mathrm{AM}$, Zaid $\mathrm{H}$, et al. A national house-staff audit of medical prophylaxis in medical patients for the PREVENTion of Venous ThromboEmbolism (PREVENT-VTE). Ir Med $J$ 2013;106:302-5. 